\title{
A RARE AND INTERESTING PRESENTATION OF LUNG TUMOUR
}

\begin{tabular}{|l|l|l|l|l|}
\hline Surgery & \\
\cline { 2 - 3 }
\end{tabular}

Dr. Sai Krishna junior resident General surgery, PG, Katuri Medical College and Hospital, Bendi* Chinakondrupadu, Guntur (District), Andhra Pradesh *Corresponding Author

\section{Dr. Raju Iyer}

\section{Dr. L. Nageswara} Rao
MS, MCh, F.C.C.P, F.I.A.C.S, (CTVS), Katuri Medical College and Hospital, Chinakondrupadu, Guntur (District), Andhra Pradesh

M.D, Anaesthesia, Katuri Medical College and Hospital, Chinakondrupadu, Guntur (District), Andhra Pradesh

\section{Dr. Satya} Narayana

M.D, Pathology, Katuri Medical College and Hospital, Chinakondrupadu, Guntur (District), Andhra Pradesh

\section{ABSTRACT}

A 72 year old male presented with a chief complaint of groin swelling and was clinically diagnosed as direct inguinal hernia and was planned for hernioplasty. Patient had no respiratory symptoms like cough or haemoptysis. Incidentally it was found that patient was having mass lesion on the left side in chest $x$ ray and was referred to CTVS department. The patient however had no complaints pertaining to the mass. He underwent CECT and was found to have ?peripheral bronchogenic tumour in left posterior mediastinum. Later left posterolateral thoracotomy was done and intra operatively mass was found originating from left lower lobe of lung and left lower lobectomy was done. Mass was around 10x15x10 cms, stony hard in consistency within the left lower lobe. The entire specimen was sent for HPE. Post operatively the patient was stable and regularly followed up. HPE of the mass was pulmonary hamartoma.

\section{KEYWORDS}

\section{Mediastinal mass 1 , Pulmonary hamartoma2}

\section{INTRODUCTION:}

Mediastinal masses ${ }^{1}$ are mostly congenital and they account for 20 $30 \%$ of all primary masses. Hamartomas ${ }^{2}$ are the most common benign lung lesions and account for more than $70 \%$ of all non-malignant tumours of lung, with peak incidence in $6^{\text {th }}$ decade of life and approximately $90 \%$ are asymptomatic

\section{CASE PRESENTATION:}

A 72 year old male from Chirala came with left groin swelling and was diagnosed as left direct inguinal hernia and planned for hernioplasty. $\mathrm{X}$ ray chest posteroanterior view was done as it is a prerequisite for pre anaesthetic check-up and a mass was found in mediastinum and referred to CTVS. Patient had no respiratory symptoms like cough, haemoptysis or shortness of breath. Previously he hadn't taken any medication. No previous history of surgeries. No similar complaints in his family. On examination he had no respiratory symptoms like cough, shortness of breath or haemoptysis.

\section{INVESTIGATIONS:}

Routine blood investigations were normal. Chest X RAY (fig. 1) showed mass in left side of mediastinum which was of homogenous opacity. CECT chest (fig. 2) showed ground glass opacity in left lower lobe. Guided biopsy showed germ cell tumour.

Fig.1 X-ray Posteroanterior View Of Chest Showing Left Mediastinal Mass

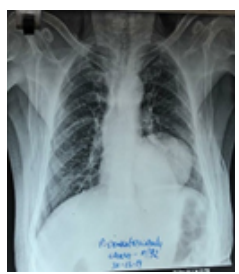

Fig . 2 CECT Chest Showing Ground Glass Opacity In Left Lower Lobe Of Lung

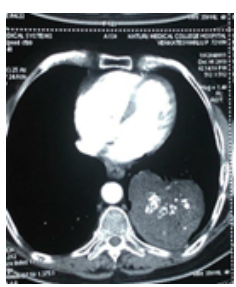

\section{PROCEDURE:}

Excision of mass done under general anaesthesia and endobronchial intubation. Left posterolateral thoracotomy was done via $6^{\text {th }}$ intercostal space, pleura opened and left lung collapsed. Tumour arising from left lower lobe was identified. Pulmonary artery branches to the left lower lobe identified and fixed. Tumour along with lower lobe isolated, bronchus to the left lower lobe clamped, fixed and sutured. No air leak, haemostasis achieved and left upper lobe ventilated. Muscles closed in two layers and specimen sent for HPE. The patient is asymptomatic and has been coming regularly for postoperative follow-up. He is scheduled for inguinal hernioplasty in the near future.

Operative findings (fig 3,4) were tumour size of $10 \times 15 \times 10 \mathrm{cms}$ occupying the left lower lobe, which was hard in consistency and well circumscribed. There was no hilar or inter lobar lymphadenopathy.

Fig.3 ,4 Showing Intra Operative Finding, Mass Of Size 10x15x10 Cms Occupying Left Lower Lobe Of Lung Which Was Well Circumscribed.

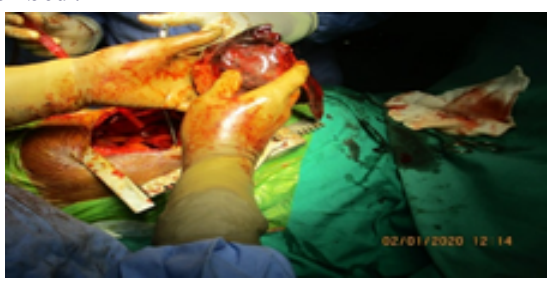

Fig.5 Showing Histological Features Of Pulmonary Chondroid Hamartoma

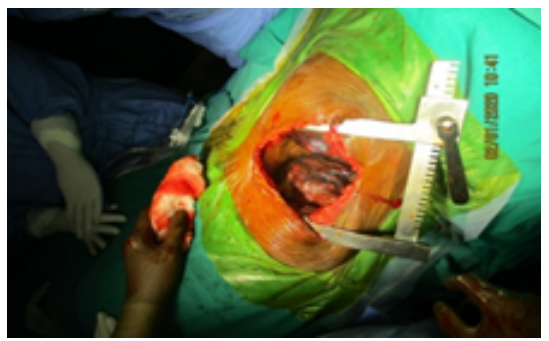

HISTOPATHOLOGY:

Fig.5 Showed histological features of pulmonary chondroid hamartoma $^{2}$ exhibiting circumscribed and uncapsulated lesion 
composed of nodules and haphazardly arranged mature hyaline cartilage admixed with mature adipose tissue, vascularised connective tissue stroma and entrapped clefts lined by respiratory lining epithelium. Focal calcification and myxomatous change seen in chondroid area.

\section{DISCUSSION:}

Hamartomas ${ }^{2}$ are the most common benign lung lesions and account for more than $70 \%$ of all non-malignant tumours of the lung. They are mesenchymal tumours with a peak incidence in the sixth decade of life, and approximately $90 \%$ are asymptomatic. Men are affected twice as often as women. About $90 \%$ of these tumours appear as solitary peripheral nodules and account for approximately $4 \%$ of all solitary pulmonary nodules.

\section{LOCATION:}

Hamartomas ${ }^{2}$ are peripheral lesions that are most often located in the lower lung fields and are well circumscribed.

\section{RADIOGRAPHIC FEATURES:}

Hamartomas $^{2}$ can be identified in chest radiograph. In CT, calcifications are described as being popcorn-like. Hamartomas display a slow growth rate and are rarely multiple. Although it is identifiable in only half of the cases, fat density as identified with CT scan is strongly suggestive of a benign hamartoma.

\section{TREATMENT:}

Surgical excision is the treatment of choice. The definitive histologic diagnosis can be only made by excision. Even in asymptomatic cases excision must be done.

\section{PATHOLOGY:}

Unlike cartilagenous tissue in normal bronchi, the cartilage in hamartomas ${ }^{2}$ tends to show irregular size, shape, and arrangement of chrondrocytes and prominent empty lacunae.

\section{CONCLUSION:}

Hamartomas $^{2}$ are the most common benign lung lesions and account for more than $70 \%$ of all non-malignant tumours of lung. Typically they are located peripherally. Surgical excision is the mainstay of treatment and can relieve all symptoms.

\section{REFERENCES :}

1. Silverman NA, Sabiston DC Jr. Mediastinal mass.Surg Clin North Am 1980;60:757-777

2. Ribet CM, Jaillard-Thery S,Nuttens MC: Pulmonary hamartoma and malignancy. J Thorax Cardiovascular Surg. 1994,107(2):611-4. 